Bond University

Research Repository

\title{
Patient Experiences of Dietary Management in Chronic Kidney Disease: A Focus Group Study
}

Kelly, Jaimon T; Campbell, Katrina L; Hoffmann, Tammy; Reidlinger, Dianne P

Published in:

Journal of Renal Nutrition

DOI:

10.1053/j.jrn.2017.07.008

Licence:

CC BY-NC-ND

Link to output in Bond University research repository.

Recommended citation(APA):

Kelly, J. T., Campbell, K. L., Hoffmann, T., \& Reidlinger, D. P. (2018). Patient Experiences of Dietary

Management in Chronic Kidney Disease: A Focus Group Study. Journal of Renal Nutrition, 28(6), 393-402. https://doi.org/10.1053/j.jrn.2017.07.008

\section{General rights}

Copyright and moral rights for the publications made accessible in the public portal are retained by the authors and/or other copyright owners and it is a condition of accessing publications that users recognise and abide by the legal requirements associated with these rights.

For more information, or if you believe that this document breaches copyright, please contact the Bond University research repository coordinator. 


\begin{abstract}
Objective: People with chronic kidney disease (CKD) contend with complex dietary recommendations. The challenge in practice is for clinicians to provide individualized support with the frequency and consistency required to sustain dietary changes. This study aimed to describe the experiences of patients with managing dietary recommendations, including their perspectives on the potential to use telehealth to support dietary management in CKD.
\end{abstract}

Design: Focus group study.

Setting: Two nephrology units in (blind) Queensland, Australia.

Subjects: 21 adult patients with CKD (non-dialysis) and 3 caregivers (total $\mathrm{N}=24$ ) purposively sampled to achieve diverse demographic and clinical characteristics.

Methods: Five focus groups were conducted, audio recorded and transcribed. Transcripts were analyzed using thematic analysis drawing on the principles of grounded theory. Main outcome measure: Themes aligned with the research question.

Results: We identified five themes: exasperating stagnancy (patronized by redundant advice, confused and unprepared for dietary change, inevitability of failure, and barriers to accessing dietetic services); supporting and sustaining change (receiving regular feedback, incremental and comprehendible modification, practical guidance on food, flexibility in monitoring schedule, and valuing peer advice); fostering ownership (seeking kidney diet information, enacting behaviour change, making reminders, and tracking progress against targets); motivators and positive learning instruction (relying on reassurance, positive reinforcement, focusing on allowable foods, and involving family); threats and ambiguities of risk (sugar as the culprit, ubiquity of salt, illegible food labelling, avoiding processed foods, and questioning credibility of sources). 
Conclusions: Patients with CKD desire a preventative approach to CKD progression and maintaining their health however are stymied by dietary restrictions and a lack of reliable dietetic advice. Easy-to-use telehealth options have the potential to overcome the shortcomings in current health service delivery which may be limiting factors to providing these approaches. They provide patients with pragmatic tools, comprehensible and consistent information which fosters ownership and self-monitoring.

Keywords: Diet; nutrition; chronic kidney disease; technology; focus groups; patientcentered care 


\section{Background}

Diet has long been considered a modifiable risk factor for chronic kidney disease (CKD), with poor dietary habits representing a key modifiable risk factor for CKD progression. ${ }^{1}$ Historically, dietary guidelines for CKD advise the restriction of individual nutrients, such as protein, sodium, phosphate and potassium. ${ }^{2}$ However, these guidelines have been criticized because they are difficult to achieve, ${ }^{3}$ and are underpinned by limited evidence for effectiveness in preventing important clinical complications including cardiovascular disease and progression to end-stage kidney disease (ESKD). ${ }^{4-7}$

Dietary advice is often multifaceted, complex and requires individualized support. ${ }^{8,9}$ Dietary and fluid restrictions can be disorienting, overwhelming, and an intense burden for patients across all stages of CKD. ${ }^{3}$ The majority of dietary interventions for pre-dialysis patients are one-off dietary education sessions, without ongoing follow up. ${ }^{10}$ In contrast, regular interaction has been shown to be a key strategy for self-management and highly valued by people with $\mathrm{CKD} .^{3}$ This mismatch in patients' needs and current clinical service provision highlights a pressing need to consider alternative strategies to support dietary change. Emerging evidence suggests that use of technology such as telehealth is effective in promoting dietary adherence, ${ }^{11,12}$ and has the potential to meet rising service demands. ${ }^{13}$ Telehealth modalities may provide an alternative framework for frequent and structured contact that is needed to support the complex dietary change required in $\mathrm{CKD}$.

There is growing recognition of the value of patient engagement in healthcare provision, and the need to focus on their experience of services, to improve efficiency and 'patient-centered care'. ${ }^{14}$ However, as far as we are aware, no study to date has focused on exploring predialysis patients' experiences of receiving CKD dietary advice, nor their preferred service modalities for accessing current healthcare services. This approach might help in the development of patient-centered interventions. The aim of this study was to describe 
experiences of patients with CKD in managing dietary recommendations, including their perspectives on the potential to use technology to support dietary management.

\section{Materials and methods}

\section{Participant selection}

Participants with CKD were recruited from two nephrology units from an urban hospital outpatient service in Queensland, Australia (blind). Eligible participants were adults aged 18 or over, had access to a mobile phone or the internet, were able to understand and speak English, not receiving dialysis treatment with CKD stage 3-4 (eGFR 15-60mL/min) and including long term-post transplant (Stage 5T). ${ }^{15}$ Long term post-transplant patients, who had a stable GFR which met our inclusion criteria were deemed eligible on the premise that their dietary management is similar as per current best-practice guidelines. ${ }^{16}$ Patients with a cognitive impairment who were unable to provide informed consent were excluded. Participants were purposively sampled to achieve diverse demographic (age, gender, and socio-demographic status) and clinical characteristics (time since diagnosis and comorbidities). Caregivers were not excluded from attending where requested by the participant in recognition of their key role in supporting the individual to self-manage and the potential insights they might provide to the research question. Participation was voluntary and written informed consent was obtained from patients and caregivers. All participants were provided with a retail gift voucher (AU\$50) to acknowledge their time. Ethical approval was granted by the (blind) Gold Coast University Hospital and Bond University Human Research Ethics Committees.

\section{Data Collection}

The focus group guide was based on a review of the literature relating to patient experience of dietary change, and use of telehealth for dietary change. We also included visual prompts 
to stimulate in-depth discussion, including an introduction to telehealth methods to all groups using photos (of interactions between patient and clinician) of telephone consultation, text messages and email communication, online workshops and videoconferencing. We also showed a YouTube video to demonstrate an example of a telehealth intervention. ${ }^{17}$ No real life examples were used in our study, and all telehealth examples discussed were hypothetical. Prior to study commencement, the topic guide was piloted with three hemodialysis patients whose results were not included in the analysis, and only used to test the depth, understanding and flow of questions. The focus groups were approximately two hours duration and conducted in a private meeting room, which provided a confidential environment. They were facilitated by the lead investigator (JK), and another researcher (DR) recorded field notes. Each group was audio recorded and later transcribed verbatim. Transcripts were de-identified and checked against the audio recording by one researcher to ensure accuracy of transcription and facilitate immersion in the data. At the conclusion of the fifth group, the authors agreed that theoretical saturation was reached (i.e. no new concepts were raised from discussions from previous focus groups).

\section{Analysis}

The transcripts were entered into NVivo 10 (QSR International, 2012) software to facilitate data analysis. Thematic analysis drawing on the principles of grounded theory was

undertaken. Two researchers (JK, DR) independently reviewed the transcript line by line to inductively identify initial codes, and through discussion and a process of constant comparison within and across focus groups, developed preliminary themes. This ensured that the preliminary analysis captured the full breadth and depth of data. A third researcher reviewed the preliminary themes and supporting quotations. Relationships among themes were developed and illustrated using a thematic schema.

\section{Results}


Twenty-four participants ( 21 patients, 3 caregivers) participated in 5 focus groups ( 3 to 8 participants per group), which were conducted between November 2015 and March 2016. Participant characteristics are provided in Table 1. Three participants were kidney transplant recipients, however still met the GFR inclusion criterion for the study.

We identified five themes: exasperating stagnancy; supporting and sustaining change; fostering ownership; motivating and positive learning; and threats and ambiguities of risk. The themes are described in detail in the following section. Selected quotations that exemplify each theme are provided in Table 2 . Figure 1 shows the conceptual links and relationship between the themes.

\section{Exasperating stagnancy}

Patronized by redundant advice - Participants believed that their priorities were misunderstood by the clinicians, including non-dietitians, who provided nutrition education. They felt patronized and given repetitive dietary advice from clinicians who would tell them the "same thing over and over and over". They believed the advice was in-actionable and "very out of date", and that they could more easily have "looked it up on the net" themselves. There was a preference for innovative dietary approaches which participants perceived to be more aligned with modern day research and that they had heard about in the media.

Confused and unprepared for dietary change - Participants felt "disheartened" and confused by the lack of specific CKD dietary advice which lacked an individualized focus. They felt unprepared and given little-to-no support on how to implement changes: "I don't know what the heck I'm doing". They also expressed frustration that they had not been prepared for potential dietary changes when they first entered into the nephrology service, "instead of learning as you go" and before their kidney condition progressed. 
Inevitability of failure - Participants described the predicament of multiple, and often conflicting, dietary recommendations for their co-morbidities. These conflicts were viewed as an impossible burden; "what in the world am I going to eat?" They expressed the view that they were being set-up to fail due to the in-cohesive approach. "[They tell you]... don't do this for that complaint and this for that complaint", and they felt "what's left over I can either eat or drink is an ice cube."

Barriers to accessing dietetic services - Participants mostly experienced healthcare consultations face-to-face. These appointments presented numerous barriers including taking time off work, cost of petrol, parking, and long waiting times. When participants were prompted on using telehealth for dietary education, they expressed that simple and familiar technology methods such as "a quick phone call or a quick text or something, or Skype" was low cost, easy and something they felt confident to use.

\section{Supporting and sustaining change}

Receiving regular feedback - Participants valued "regular contact" and "a bit of feedback" to track their progress as they saw it supporting their dietary change over time. They desired regular, more intense follow up in the early stages of modifying diet "just until you got your plan under way and under control" which could "spin out a bit more" (become less intensive) when their confidence increased. They highlighted the importance of the time between feedback being "individually tailored" to their condition and transitioning life circumstances. Incremental and comprehendible modification - Participants stressed the need for dietary change to be taken "one footstep" at a time. They described their experience of instructional and overwhelming advice as like a "rubber-stamp on the forehead". They suggested technology might play a complementary role to face to face contact through methods such as 
SMS and/or telephone follow up to highlight the "basic stuff [diet education] first" and then "further detail as you go along".

Practical guidance on food - Many participants wanted a "list [or] guideline of what foods you could have... and what to leave out and maybe what to have occasionally". Participants favored "the practical advice... you don't hear that very often from anybody". They wanted focused practical food-based advice to help them make sense of the nutrient focused information they were given previously.

Flexibility in monitoring schedule - Participants wanted the power to decide their own monitoring schedule. They wanted this to be two-way with "the option to ring the dietitian". While they highly valued regular review, they believed increasing review appointments would add to their existing and competing problems accessing current healthcare. They were interested in how technology consultations could give them flexibility including the option to request that clinicians "ring you another day".

Valuing peer advice - Many participants had never spoken about dietary challenges with other people with CKD. They thought the strategies and advice from peer support groups "sticks in your mind more, [and allows you to] cross-fertilize ideas", but were unaware of group services available to them. Peer advice from dialysis patients was highly valued, and many described the prospect of dialysis as "scary", something they wanted to avoid. They preferred to talk to their peers who were already on dialysis because they "knew what it was about" in a way that a clinician could not.

\section{Motivating and positive learning instruction}

Relying on reassurance - The potential nurturing role of the clinician was highly valued. They wanted to "feel reassured, [to] ask questions [and get a sense of] I'm okay". Part of "feeling reassured [was knowing] that things can be done to improve [or] stabilize" their 
kidney problems. They felt they currently did not have an ongoing supportive partnership with their healthcare provider. They saw telehealth as one method to provide reassurance, to complement face-to-face, such as "a text saying 'you're okay', that's terrific feedback".

Positive reinforcement - Participants talked about both positive and negative experiences of clinicians giving them dietary advice. They preferred advice that was respectful, and "never... put you down" which they believed reinforced the changes they had to make. They avoided punitive and instructive approaches such as "you eat this", "you stick to this". Clinicians that were "gentle" and "friendly" were viewed positively and they felt more comfortable, and happy to listen to dietary advice from them.

Focusing on allowable foods - Participants felt a CKD diet was very restrictive. They wanted information on "what foods you could have" rather than being told what nutrients to avoid. They described feeling "disheartened" by dietary restrictions which were viewed as "pretty severe”. Because they didn't know what they were allowed to eat, they talked of using technology, such as the internet, however this wasn’t always successful; "all they [internet sources] seem to list is high potassium foods" (and not the low potassium foods allowed). Involving family - Participants felt that family and caregivers were gatekeepers to change. They discussed the importance of involving family members or friends, describing how they "regulate" their diets. They saw dietary advice as "a two-person-thing", which should include both "the cook and the patient". If family were not involved in dietary decisions or lacked understanding, they described having to prepare separate meals; "I can't feed anyone else the same stuff as I eat... you've got to take into consideration what they're going to eat".

\section{Fostering ownership}

Seeking kidney diet information - Participants described how they liked "picking up stuff off the internet", specifically for information not well explained by their clinician. Other 
participants sought answers through sources like "Google Scholar", "books", "newsletter" and "the news" in a quest to self-educate on dietary change. They felt that their doctors "don't have time" to answer all their questions, and favored "instant" and "non-invasive" methods to seek their information, such as the telephone and text messaging services. Enacting behaviour change - When prompted to the types of dietary education commonly provided to patients with CKD, participants believed "a dietitian's got that stuff [dietary information] already out there". They argued that achieving dietary behaviour changes requires them as individuals to "want ownership of the problem", to "tell them [the dietitian] how I'm going myself" and drive their own change "if you want it, if you need it".

Making reminders - Participants discussed a desire "to get a sort of a reminder" as a tool to improve their own self-monitoring and efficacy. Participants discussed the importance of being reminded about their recommendations for dietary change, but also their clinic appointments. This included a reminder of dietary education delivered by phone or face-toface; "how about a reminder about the call they were going to receive?"; or a text saying "I called you the other day, how are you going?".

Tracking progress against targets - Participants preferred to "track" the results of their blood tests to "physically see" the progress made. They discussed that they often ask their doctor "what's my readings?" for reassurance that they were "heading in the right direction", and this was a motivator to make dietary adjustments. Participants suggested record keeping methods using technology so it was possible to track "the results of your last blood test" by using the internet and mobile apps such as "Carb Count [mobile app] or something like that".

\section{Threats and ambiguities of risk}

Sugar as the culprit-All participants described sugar as "poison", believing "white [sugar] is death" and it was a very important dietary belief to protect their kidneys from further damage. 
Many participants were of the opinion that 'brown' and 'raw' sugars were healthier choices and would use these in place of white sugar, whereas others "just stop sugar full stop". Participants discussed many challenges to finding suitable "substitutes that is going to be interesting or tasty" in place of regular sugar.

Ubiquity of salt - Participants discussed that there "seem to be a lot of things on the market that have salt in them". They described it as "pretty frustrating" and impossible to "find anything that hasn't got any salt in it". While most participants were very "conscious of my salt", some thought "it's not good if you're without salt... your body needs the salt".

Illegible food labelling - Many participants recognized label reading as important to make better food choices. They faced the dilemma of identifying a low sugar product, only to find it was high in salt. They believed food "labels should be changed" particularly regarding their size and format to "be a decent legible size". Many participants required glasses to read labels, and were frustrated about taking "twice as long to do the grocery shopping".

Avoiding processed foods - Participants reported a common belief that the food supply was dominated by processed foods. They reported having "trouble" with avoiding "all the processed stuff, which is really difficult to do without these days". They discussed what would "be the best [would be] if some of the processed food had less salt [and weren't] full of preservatives" which they described made it "so hard" to rationalize what to prioritize.

Questioning credibility of sources - Participants were seeing many different healthcare professionals, and regularly received new and sometimes conflicting advice. Those who frequently sought out information themselves described the need to "check with somebody that knows what they're talking about" to question the reliability of information they found, particularly if it was food related and something they had not been previously advised about. They discussed how they would "research something [on the internet] and know that I could 
be possibly getting the wrong information [so they would] print it out and run it by the doctor". 


\section{Discussion}

This qualitative study explored the experiences of CKD participants in relation to dietary management, and their perspectives on the potential for telehealth to support dietary change. Participants in our study described feeling misunderstood by clinicians providing dietary advice, and felt confused with conflicting guidance from different clinicians. In addition, they highlighted many barriers to seeing clinicians in face-to-face settings, which did not meet their preference for regular review and feedback. Participants desired practical guidance on foods and a flexible monitoring schedule to support and sustain dietary change. They preferred clinicians who provided reassurance, were positive, involved their family and/or caregivers, and focused on foods that were allowed in the diet, as opposed to imposing food restrictions. To gain control of their diets, they felt responsible for seeking out additional dietary information, enacting dietary changes and self-tracking their progress over time. This was an exceptional challenge for participants whose health care delivery experience was not conducive to making dietary behaviour change.

This is the first qualitative study to describe the CKD patient experience with dietary advice provided in nephrology services. Although we know that patients struggle with adhering to their diet and fluid recommendations, and desire more flexibility and individualised support, ${ }^{3}$ our study has focused on the source of these recommendations as well as their delivery setting. For the first time, we show that diet recommendations come from a myriad of sources (and rarely from qualified dietitians). People with CKD find this a disorienting and confusing experience. Our study suggests that telehealth may be viable options to overcome these patient-centered care barriers. Importantly, patients did not show preference for what telehealth options would be 'the best', as long as these were cheap, easy to use, and gave them flexibility. We believe this will help future intervention development and continued efforts to improve patient-centered care in nephrology services. 
Participants felt their priorities for dietary change were neglected by the wider healthcare community (including primary care and nephrology services). They described an inflexible, outdated, and patronizing consumer experience, not aligned to patient-centered care. Such shortcomings in delivering patient-centered care in nephrology services has been reported previously ${ }^{18,19}$ and is increasingly recognized as a barrier to delivering effective clinical care, to improve patient-clinician communication and overall health outcomes. ${ }^{20}$

The findings of this study highlight that people with CKD want advice and greater emphasis on prevention and progression of CKD in the early stages. Therefore, a coordinated model of care fostering ownership over their dietary self-management and supporting change longterm is needed. General practitioners and nephrologists are best placed to initially highlight the importance of diet to newly diagnosed patients, and to refer to a dietitian shortly after diagnosis. A coordinated and multidisciplinary approach to patient care has been shown to improve patient outcomes, ${ }^{21}$ is a strong service need and reflects patient's desires. There is evidence to support healthy dietary change in $\mathrm{CKD}$, including the reduction of dietary sodium for lowering blood pressure and proteinuria, ${ }^{4}$ and protein modification for protecting residual kidney function. ${ }^{22}$ Furthermore, the adoption of a healthy dietary pattern may reduce incident CKD in the general population ${ }^{23}$ and mortality in established kidney disease. ${ }^{24}$ Yet despite an evidence-base for prevention, this patient population feel unable to access dietary services, at a time they are motivated to prevent their disease progression.

The current healthcare workforce and models of care appear unable to meet patient expectations of patient-centered care. With current service delivery unable to adjust to increasing consumer demand, ${ }^{25}$ many patients will never see a renal dietitian. Instead, patients may only receive these services if they reach ESKD, where they may be referred to manage acute concerns, such as hyperkalemia. However, ESKD is the patient-centered endpoint they describe being desperate to avoid. While services continue to neglect this 
prevention focus, patients perceive stagnancy in healthcare delivery, are innately confused, and feel unprepared to make dietary change. This can impede patients' self-efficacy for integrating dietary self-management, leaving them to seek diet information online which often lacks evidence and may cause harm. ${ }^{26}$ If current service models cannot address people's self-efficacy in the early stages of CKD, this may lead to faster progression to ESKD. ${ }^{27}$ Using telehealth shows considerable promise for improving self-efficacy in people with CKD, and may overcome current healthcare limitations and improve patient-centered care. Our group of CKD participants expressed interest in telehealth methods that would complement their traditional care rather than replace it, providing the delivery modality was relevant and familiar to their skills and level of health literacy. Participants preference for telehealth primarily centered on overcoming the incoherent dietary advice in current health service delivery. Many participants were already using mobile devices and the internet, particularly for seeking information outside of their usual care, therefore these modalities were often preferred and acceptable to support self-mangement. ${ }^{28}$ These methods were seen as ways 'Receive regular feedback', having 'Flexibility in the monitoring schedule', able to 'Involve family', and overall 'Foster ownership'; while overcoming the growing 'Barriers to accessing dietetic services'. Using telehealth has been shown to facilitate dietary change ${ }^{12}$ and could overcome the common healthcare barriers experienced in face-to-face settings, including demographic isolation, working hours, and forgetting appointments. ${ }^{29}$ Additionally, telehealth methods could decrease commonly reported healthcare access barriers, specifically administrative errors, access to clinic facilities, parking and unfavorable opening hours of clinics in face-to-face settings. ${ }^{19,30}$

It is important to note that telehealth may introduce the opportunity for health services to utilize non-dietary trained personnel in its delivery. However, the results of this study indicate that the majority of participants had never seen a renal dietitian, leading to many of 
their dietary challenges, and the health service perceived as stagnant and unsupportive. Based on these findings, we recommend any telehealth dietary intervention should involve a dietitian, at least in the development of materials (text messages; call scripts; resources) and preferably in the subsequent delivery of the intervention content. Given telehealth's novelty in $\mathrm{CKD}$, any program should be pre-tested with the patient demographic and ideally trialed for feasibility and cost-effectiveness prior to implementation.

As far as we are aware, this is the first study to describe the experiences of people's dietary management in pre-dialysis CKD and perspectives on the potential to use telehealth to support dietary change. The use of a semi-structured questioning approach, to encourage participants to discuss their feelings and attitudes (both positive and negative) was a strength which somewhat mitigates the inherent researcher bias. However, this study is not without limitations. Given its qualitative design, the generalizability of themes to other populations with CKD is uncertain. Although we attempted to achieve a demographic reflective of current prevalence in Australia, we did not recruit participants of Indigenous heritage, or those living outside metropolitan areas. However, the results are generally consistent with similar ESKD qualitative studies. ${ }^{3}$ Future research should specifically target these populations, who are known to have higher burden of CKD and who may experience different barriers to dietary change and technology use.

In summary, people with pre-dialysis CKD desire a preventative approach to $\mathrm{CKD}$ progression however, are stymied by restriction-focused dietary advice and a lack of dietetic service provision in early stage CKD. Clinicians need to be positive and motivating, and consider the individual's goals for dietary change, to empower patients to better cope with dietary recommendations, which could help improve patient-centered outcomes. Telehealth methods such as the telephone and text messaging were viewed acceptable over other telehealth options (and to complement face to face care) to facilitate dietary follow up. These 
modalities appear to meet the patients' expectations of service delivery, and may assist in dietary change.

\section{Practical application}

Patients with CKD experience a range of barriers to enacting dietary behaviour change. These partly stem from the characteristics of health professionals and barriers to traditional healthcare delivery. Clinicians should align with a patient's priority for dietary change, and avoid patronizing advice focused on nutrient restrictions. This patient-engagement study can be used to develop patient-centered telehealth programs, and guide its implementation in a CKD service. To use telehealth to support dietary change, clinicians should consider text messages and phone calls, as they are viewed to facilitate regular, repeated interaction and giving patients the support they require for long-term dietary change. 


\section{References}

1. National Kidney Foundation. KDOQI Clinical Practice Guidelines and Clinical Practice Recommendations for Diabetes and Chronic Kidney Disease. American journal of kidney diseases : the official journal of the National Kidney Foundation. 2007;49(2 Suppl 2):S1-S180.

2. Levey AS, Rocco MV, Anderson S, et al. K/DOQI clinical practice guidelines on hypertension and antihypertensive agents in chronic kidney disease. American Journal of Kidney Diseases. 2004;43(5 SUPPL. 1).

3. Palmer SC, Hanson CS, Craig JC, et al. Dietary and Fluid Restrictions in CKD: A Thematic Synthesis of Patient Views From Qualitative Studies. American journal of kidney diseases : the official journal of the National Kidney Foundation. 2014;65(4):559-573.

4. McMahon EJ, Campbell KL, Bauer JD, Mudge DW. Altered dietary salt intake for people with chronic kidney disease. Cochrane Database of Systematic Reviews. 2015;2:Cd010070.

5. Liu Z, Su G, Guo X, et al. Dietary interventions for mineral and bone disorder in people with chronic kidney disease. The Cochrane database of systematic reviews. 2015.

6. Fouque D, Laville $M$, Boissel J. Low protein diets for chronic kidney disease in non diabetic adults. Cochrane Database of Systematic Reviews. 2009;3.

7. Kotwal S, Jun M, Sullivan D, Perkovic V, Neal B. Omega 3 fatty acids and cardiovascular outcomes. Systematic review and meta-analysis. Circ Cardiovasc Qual Outcomes. 2012; 5: 808-18. External Resources Pubmed/Medline (NLM) CrossRef (DOI). 2016.

8. Smyth A, O'Donnell MJ, Yusuf $S$, et al. Sodium intake and renal outcomes: a systematic review. Am J Hypertens. 2014;27(10):1277-1284.

9. Mozaffarian D. Dietary and Policy Priorities for Cardiovascular Disease, Diabetes, and Obesity A Comprehensive Review. Circulation. 2016;133(2):187-225.

10. St Peter WL, Schoolwerth AC, McGowan T, McClellan WM. Chronic kidney disease: issues and establishing programs and clinics for improved patient outcomes. American journal of kidney diseases. 2003;41(5):903-924.

11. Desroches S, Lapointe A, Ratte S, Gravel K, Legare F, Turcotte S. Interventions to enhance adherence to dietary advice for preventing and managing chronic diseases in adults. The Cochrane database of systematic reviews. 2013;2:Cd008722.

12. Kelly JT, Reidlinger DP, Hoffmann TC, Campbell KL. Telehealth methods to deliver dietary interventions in adults with chronic disease: a systematic review and metaanalysis. The American Journal of Clinical Nutrition. 2016;104(6):1693-1702.

13. Daly R. Transitioning health systems for multimorbidity. Transplantation. 2012;93:348-353.

14. Australian Commission on Safety and Quality in Health Care. Patient-Centred Care: Improving Quality and Safety by Focusing Care on Patients and Consumers Discussion Paper. In: Care ACoSaQiH, ed. Australia2010.

15. Eknoyan G, Lameire N, Eckardt KU, et al. KDIGO 2012 clinical practice guideline for the evaluation and management of chronic kidney disease. Kidney Int. 2013;3:5-14. 
16. Chan M, Patwardhan A, Ryan C, et al. Evidence-based guidelines for the nutritional management of adult kidney transplant recipients. Journal of Renal Nutrition. 2011;21(1):47-51.

17. YouTube. - Looking up your results in PatientView. [Cited 18 Jan 2016.]; https://www.youtube.com/watch?v=Ri1wXrCGH9c\&index=2\&list=PLGk58HmEpP7II M1wP1FlbpGxEfEaxO-Ft

18. Bear RA, Stockie S. Patient engagement and patient-centred care in the management of advanced chronic kidney disease and chronic kidney failure. Canadian journal of kidney health and disease. 2014;1(1):1.

19. Lo $\mathrm{C}$, llic D, Teede $\mathrm{H}$, et al. The Perspectives of Patients on Health-Care for Co-Morbid Diabetes and Chronic Kidney Disease: A Qualitative Study. PloS one. 2016;11(1).

20. Mead N, Bower P. Patient-centred consultations and outcomes in primary care: a review of the literature. Patient education and counseling. 2002;48(1):51-61.

21. Goldstein M, Yassa T, Dacouris N, McFarlane P. Multidisciplinary predialysis care and morbidity and mortality of patients on dialysis. American Journal of Kidney Diseases. 2004;44(4):706-714.

22. Kasiske BL, Lakatua J, Ma JZ, Louis TA. A meta-analysis of the effects of dietary protein restriction on the rate of decline in renal function. American Journal of Kidney Diseases. 1998;31(6):954-961.

23. Smyth A, Griffin M, Yusuf S, et al. Diet and Major Renal Outcomes: A Prospective Cohort Study. The NIH-AARP Diet and Health Study. Journal of Renal Nutrition. 2016;26(5):288-298.

24. Kelly J, Campbell K, Wai S, et al. Healthy Eating Patterns, Mortality and End-Stage Kidney Disease in CKD: A Systematic Review and Meta-Analysis. Clin J Am Soc Neprol. 2016;12(2):272-279.

25. Campbell KL, Murray EM. Allied health services to nephrology: an audit of current workforce and meeting future challenges. J Ren Care. 2013;39(1):52-61.

26. Ande P, Chiu D, Rayner S, Coward RA, Woywodt A. What's on the web for nephrology? NDT plus. 2009;2(2):119-126.

27. Curtin RB, Walters BA, Schatell D, Pennell P, Wise M, Klicko K. Self-efficacy and selfmanagement behaviors in patients with chronic kidney disease. Advances in chronic kidney disease. 2008;15(2):191-205.

28. Cueto-Manzano AM, Gallardo-Rincón H, Martínez-Ramírez HR, et al. A pilot study of a mobile phone application to improve lifestyle and adherence of patients with kidney disease. Journal of telemedicine and telecare. 2015;21(2):119-120.

29. Murdock A, Rodgers C, Lindsay $H$, Tham TCK. Why do patients not keep their appointments? Prospective study in a gastroenterology outpatient clinic. J Royal Soc Med. 2002;95(6):284-286.

30. Paterson BL, Charlton P, Richard S. Non - attendance in chronic disease clinics: a matter of non - compliance? Journal of Nursing and Healthcare of Chronic IIIness. 2010;2(1):63-74. 


\section{Tables}

Table 1. Chronic Kidney Disease Participant characteristics $(\mathrm{n}=21)$

Table 2. Selected quotes for each sub-theme within the five key themes developed 


\section{Figures}

Figure 1. Thematic schema of patients' perspectives of dietary recommendations and telehealth. The dotted lines represent supporters and solid lines represent inhibitors pertaining to each theme. The top circles represent patient-centered characteristics, and the bottom circles clinician and health service characteristics. 